\title{
Classical and Bayesian methods of estimation for power Lindley distribution with application to waiting time data
}

\author{
Vikas Kumar Sharma ${ }^{1, a}$, Sanjay Kumar Singh ${ }^{b}$, Umesh Singh $^{b}$ \\ ${ }^{a}$ Department of Mathematics, IITRAM, India; \\ ${ }^{b}$ Department of Statistics and DST-CIMS, Banaras Hindu University, India
}

\begin{abstract}
The power Lindley distribution with some of its properties is considered in this article. Maximum likelihood, least squares, maximum product spacings, and Bayes estimators are proposed to estimate all the unknown parameters of the power Lindley distribution. Lindley's approximation and Markov chain Monte Carlo techniques are utilized for Bayesian calculations since posterior distribution cannot be reduced to standard distribution. The performances of the proposed estimators are compared based on simulated samples. The waiting times of research articles to be accepted in statistical journals are fitted to the power Lindley distribution with other competing distributions. Chi-square statistic, Kolmogorov-Smirnov statistic, Akaike information criterion and Bayesian information criterion are used to access goodness-of-fit. It was found that the power Lindley distribution gives a better fit for the data than other distributions.
\end{abstract}

Keywords: power Lindley distribution, maximum likelihood estimator, least squares estimator, maximum product spacings estimator, Bayes estimator, goodness-of-fit test

\section{Introduction}

Lindley distribution indexed by a scale parameter was first introduced by Lindley (1958) in the context of Bayesian theory, as a counter example of fiducial statistics. Ghitany et al. (2008) discussed various statistical and mathematical properties of the Lindley distribution in order to advocate its use as a lifetime model over exponential distribution. Subsequently, the Lindley distribution has been used by many authors in modelling lifetime data under different scenarios, see Ali et al. (2013), Al-Mutairi et al. (2013), Krishna and Kumar (2011), Sharma et al. (2016a), and references cited therein. The probability density function (pdf) of the Lindley distribution is given by

$$
f(x ; \beta)=\frac{\beta^{2}}{1+\beta}(1+x) e^{-\beta x}, \quad x>0, \beta>0 .
$$

The Lindley distribution is inadequate to model some real phenomenon since it is not much flexible to model various skewed data sets with a scale parameter only. In this view, Gupta and Kundu (2009) discussed the importance of the shape parameter in modelling and gave comprehensive review on different methods to introduce a shape/skewness parameter in an arbitrary probability distribution. Two methods namely exponentiation and power transformation are important choices among others and used to develop more flexible distribution with an added shape parameter. In the first method,

\footnotetext{
${ }^{1}$ Corresponding author: Department of Mathematics, Institute of Infrastructure, Technology, Research and Management (IITRAM), Ahmedabad, India. E-mail: vikasstats@rediffmail.com
}

Published 31 May 2017 / journal homepage: http://csam.or.kr

(c) 2017 The Korean Statistical Society, and Korean International Statistical Society. All rights reserved. 
a constant as a power of the cumulative distribution function (cdf) of a baseline distribution is introduced, i.e., $F(x)=[G(x)]^{\alpha}$, where $G(x)$ is the baseline distribution function and $\alpha(>0)$ is the proportionality constant. The models obtained are called proportional reverse hazard rate models. In the second method, we apply a power transformation to the given random variable i.e., $Y=X^{\alpha}$, where $Y$ is the baseline random variable. Weibull distribution (WD) is a good example of a power transformed model for exponential distribution. Using these approaches, two extensions of the Lindley distribution known as power Lindley distribution (PLD) and generalized Lindley distribution (GLD) are proposed by Ghitany et al. (2013) and Nadarajah et al. (2011), respectively. A random variable $X=Y^{1 / \alpha}$ is said to follow the PLD, if $Y$ follows the Lindley distribution. The pdfs of GLD and PLD are respectively given by

$$
\begin{aligned}
& f_{G}(x ; \alpha, \beta)=\frac{\alpha \beta^{2}(1+x)}{1+\beta}\left(1-\frac{1+\beta+\beta x}{1+\beta} e^{-\beta x}\right)^{\alpha-1} e^{-\beta x}, \quad x>0, \beta>0, \alpha>0, \\
& f_{P}(x ; \alpha, \beta)=\frac{\alpha \beta^{2}}{1+\beta}\left(1+x^{\alpha}\right) x^{\alpha-1} e^{-\beta x^{\alpha}}, \quad x>0, \beta>0, \alpha>0 .
\end{aligned}
$$

The GLD is considered by many authors under various contexts. Singh et al. (2013, 2014b) discussed Bayes estimation of parameters of the GLD in presence of complete and progressively censored samples respectively. Okwuokenye and Peace (2015) compared two GLD based on the size and power of the test of hypotheses on parameters. Gui and Chen (2016) developed inverse and modified inverse moment estimators of the GLD parameters.

The PLD is quite flexible to accommodate common ageing classes of increasing, decreasing and decreasing-increasing-decreasing hazard rates. Ghitany et al. (2013) illustrated the applicability of the PLD through real data modelling and showed that PLD fits better than other two-parameter distributions such as gamma, Weibull, Gompertz. In their work, Ghitany et al. (2015) discussed the maximum likelihood estimation (MLE) for strength-stress reliability based on a complete sample of observations from PLD. Singh et al. (2014a) discussed MLE as well as Bayes estimation under hybrid censoring schemes. PLD has been given less attention despite it being a parsimonious model with nice properties such as closed form distribution and survival functions and flexible shapes of pdf and hazard functions as well as being a good competitor of WD and gamma distributions (GD). Therefore, this article discusses various classical and Bayes estimators to estimate unknown parameters of PLD and its application to waiting time data.

In the lieu of the above considerations, we discuss MLE, least squares estimator (LSE), maximum product spacings (MPS), and Bayes estimation methods for the PLD and studied their behaviors with respect to varying sample size and various combinations of parameter values. Both noninformative and informative (gamma) priors are used for Bayesian estimation. Bayes estimators are not in closed forms; therefore, we propose the use of Lindley approximation and Markov chain Monte Carlo (MCMC) techniques for Bayesian calculations.

The rest of the paper has been organized in the following sections. In Section 2, some properties of PLD are derived. Classical and Bayes estimators of PLD parameters are constructed in Section 3 and 4, respectively. In Section 5, the proposed estimators are compared using simulated samples from PLD. In Section 6, two data sets on waiting times to accept research articles for publication in statistics journals noted from volumes published in the 2012 of Journal of Statistical Planning and Inference (JSPI) and Computational Statistics and Data Analysis (CSDA) are fitted to various statistical distributions and then analyzed with the discussed procedures. Finally, the conclusions are given in Section 7. 


\section{Some properties of power Lindley distribution (PLD)}

\subsection{Moments and mean/median deviation}

The $r^{\text {th }}$ moment, derived by Ghitany et al. (2013), for the PLD is given by

$$
E\left(X^{r}\right)=\frac{\alpha \beta^{2}}{\beta+1} \frac{r \Gamma(r / \alpha)(\alpha(\beta+1)+r)}{\alpha^{3} \beta^{\frac{r}{\alpha}+2}} .
$$

Mean deviation (MD) is the expected value of the absolute deviation of $X$ about $A, E_{f_{x}}[X-A]=$ $\mathrm{MD}_{A}$. It measures the scatteredness of a distribution function about an arbitrary number $A$. Its value is large when there is a great scattering and small when there is a great concentration about $A$. MD is generally taken about the mean; however, it is least when taken about the median. MD about mean $(\bar{X})$ and median $(\tilde{X})$ can be obtained as,

$$
\begin{aligned}
\operatorname{MD}_{\bar{X}} & =\int_{0}^{\infty}|x-\bar{X}| d F(x)=-2 \bar{X}\left(1+\frac{\beta}{1+\beta} \bar{X}^{\alpha}\right) e^{-\beta \bar{X}^{\alpha}}+2 \frac{\alpha \beta^{2}}{\beta+1} I(\alpha, \beta, 1, \bar{X}), \\
\operatorname{MD}_{\tilde{X}} & =\int_{0}^{\infty}|x-\tilde{X}| d F(x)=-\bar{X}+2 \frac{\alpha \beta^{2}}{\beta+1} I(\alpha, \beta, 1, \tilde{X}),
\end{aligned}
$$

where $I(\cdot, \cdot, \cdot, \cdot)$ is given by Lemma 1 in Appendix.

\subsection{Conditional moments}

The $r^{\text {th }}$ conditional moment can be defined as

$$
E\left(X^{r} \mid X>x\right)=\frac{1}{P(X>x)} \int_{x}^{\infty} x^{r} f(x) d x=\frac{1}{1-F(x)} \frac{\alpha \beta^{2}}{1+\beta} I(\alpha, \beta, r, x),
$$

where $F(\cdot)$ is cdf of the PLD. However, the mean residual lifetime function is $E\left(X^{1} \mid X>x\right)-x$. The mean residual life function plays an important role in many fields such as engineering, medicine, life insurance, and demographics. The references from Abdous and Berred (2005), Govil and Aggarwal (1983) provide more details and application. The mean residual life function is defined as

$$
e(x)=E(X-x \mid X>x)=\frac{1}{1-F(x)} \int_{x}^{\infty} 1-F(x) d x .
$$

On some simplifications, we get

$$
e(x)=\frac{(1+\beta) \Gamma\left(1 / \alpha, \beta x^{\alpha}\right)+\Gamma\left((\alpha+1) / \alpha, \beta x^{\alpha}\right)}{\alpha \beta^{\frac{1}{\alpha}}\left(1+\beta+\beta x^{\alpha}\right) e^{-\beta x^{\alpha}}},
$$

where $\Gamma(a, x)=\int_{x}^{\infty} y^{a-1} e^{-y} d y$ is the complimentary incomplete gamma function.

\subsection{Moment generating function}

The moment generating function is simply defined

$$
M_{x}(t)=\frac{\alpha \beta^{2}}{1+\beta} \int_{0}^{\infty} x^{\alpha-1} e^{-\beta x^{\alpha}+t x} d x+\frac{\alpha \beta^{2}}{1+\beta} \int_{0}^{\infty} x^{\alpha} e^{-\beta x^{\alpha}+t x} d x .
$$


By Lemma 2 (Appendix), we have

$$
\begin{aligned}
M_{x}(t) & =\frac{\alpha \beta^{2}}{1+\beta}[G(\alpha, \beta,-t, \alpha, 1)+G(\alpha+1, \beta,-t, \alpha, 1)] \\
& =\frac{1}{\beta^{\frac{1}{\alpha}-1}(1+\beta)}\left[H_{0,2}^{2,0}\left[-\left.t \beta^{\frac{1}{\alpha}}\right|_{(0,1)\left(1, \frac{1}{\alpha}\right)}\right]+H_{0,2}^{2,0}\left[-\left.t \beta^{\frac{1}{\alpha}}\right|_{(0,1)\left(\frac{1}{\alpha}+1, \frac{1}{\alpha}\right)}\right]\right] .
\end{aligned}
$$

\subsection{Entropy measures}

The entropy measure is used to measure the amount of uncertainty associated with a random variable $X$ entertained in scientific fields. The most popular entropy measures are Rényi entropy and Shannon entropy. The Rényi entropy is defined as

$$
I_{R}=\frac{1}{1-\gamma} \log \left[\int f^{\gamma}(x) d x\right], \quad \gamma>0(\neq 1) .
$$

Substituting the pdf of PLD in above equation, we obtain

$$
I_{R}=\frac{1}{1-\gamma} \log \left[\frac{e^{\beta \gamma}}{\alpha \beta \gamma} \sum_{k=0}^{\infty}(-1)^{k}\left(\begin{array}{c}
(\alpha \gamma-\gamma-\alpha+1) / \alpha \\
k
\end{array}\right) \frac{\Gamma((2 \alpha \gamma-\gamma-k \alpha+1) / \alpha, \beta \gamma)}{(\beta \gamma)^{(2 \alpha \gamma-\gamma-\alpha+1) / \alpha-k}}\right] .
$$

Shannon's entropy is defined as $I_{S}=-E_{f}[\log (f(x))]=-\int_{0}^{\infty} \log (f(x)) f(x) d x$. On simplifications, we get

$$
I_{S}=-\log \left[\frac{\alpha \beta^{2}}{1+\beta}\right]-\frac{e^{\beta}}{1+\beta}\left[e^{\beta}+E_{1}(\beta)\right]+\frac{2+\beta}{1+\beta}-(\alpha-1) \frac{\beta^{2}}{1+\beta}[I(1, \beta)+I(2, \beta)],
$$

where $E_{n}(z)=\int_{1}^{\infty}\left(e^{-z t} / t^{n}\right) d t$ is the exponential integral and $I(a, b)$ is defined by Lemma 3 in Appendix.

\section{Classical estimation of the parameters}

\subsection{Estimation based on likelihood}

Let $x_{1}, x_{2}, \ldots, x_{n}$ be an independent and identical random sample of size $n$ from the PLD (1.3). The log-likelihood function based on observed sample $\underline{x}=\left\{x_{1}, x_{2}, \ldots, x_{n}\right\}$ is given by

$$
\log L=n \ln (\alpha)+2 n \ln (\beta)-n \ln (1+\beta)+\sum_{i=1}^{n} \ln \left(1+x_{i}^{\alpha}\right)+(\alpha-1) \sum_{i=1}^{n} \ln \left(x_{i}\right)-\beta \sum_{i=1}^{n} x_{i}^{\alpha} .
$$

The MLEs $\hat{\alpha}$ and $\hat{\beta}$ of $\alpha$ and $\beta$, respectively, can be obtained as the simultaneous solution of the following two non-linear equations:

$$
\begin{aligned}
& \frac{\alpha}{n}+\sum_{i=1}^{n} \frac{x_{i}^{\alpha} \ln \left(x_{i}\right)}{1+x_{i}^{\alpha}}+\sum_{i=1}^{n} \ln \left(x_{i}\right)-\beta \sum_{i=1}^{n} x_{i}^{\alpha} \ln \left(x_{i}\right)=0, \\
& \frac{n(\beta+2)}{\beta(\beta+1)}-\sum_{i=1}^{n} x_{i}^{\alpha}=0 .
\end{aligned}
$$



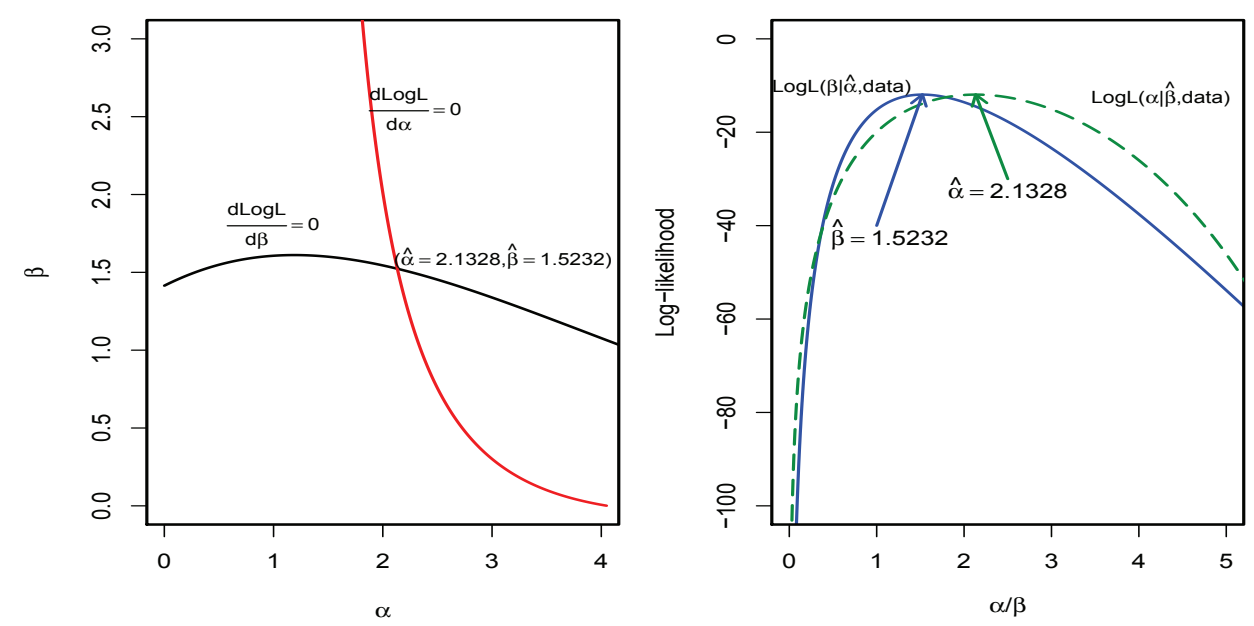

Figure 1: Plots of likelihood equations (left) and likelihood profile (right) for $\alpha$ and $\beta$.

By solving the quadratic equation (3.5) in $\beta$, the MLE $\hat{\beta}$ of $\beta$ can be uniquely determined as follows,

$$
\hat{\beta}(\hat{\alpha})=\frac{-\left(\sum_{i=1}^{n} x_{i}^{\hat{\alpha}}-n\right)+\sqrt{\left(\sum_{i=1}^{n} x_{i}^{\hat{\alpha}}-n\right)^{2}+8 n \sum_{i=1}^{n} x_{i}^{\hat{\alpha}}}}{2 \sum_{i=1}^{n} x_{i}^{\hat{\alpha}}},
$$

where $\hat{\alpha}$ is the solution of the following equation

$$
\frac{\alpha}{n}+\sum_{i=1}^{n} \frac{x_{i}^{\alpha} \ln \left(x_{i}\right)}{1+x_{i}^{\alpha}}+\sum_{i=1}^{n} \ln \left(x_{i}\right)-\hat{\beta}(\hat{\alpha}) \sum_{i=1}^{n} x_{i}^{\alpha} \ln \left(x_{i}\right)=0 .
$$

In order to solve above equation, one can apply a suitable iterative procedure such as the NewtonRaphson method.

\subsubsection{Existence and uniqueness of the MLEs}

It is of interest to show the existence and uniqueness of the MLEs. Its analytical demonstration is complicated since Fisher's information includes intractable integrals. Therefore, we investigate the existence and uniqueness of the MLEs numerically based on the superimposed curves of loglikelihood equations. The steps that we followed are:

Step 1. Without loss of generality, we simulate a random sample of size 25 from the PLD with $\alpha=2$ and $\beta=2$.

Step 2. The curves of the $\log$-likelihood equations, $d \log L / d \alpha=0$ and $d \log L / d \beta=0$ for $\alpha$ and $\beta$ are sketched in Figure 1.

Step 3. From Figure 1, it can be seen that there exists a intersection point $(\hat{\alpha}=2.1328, \hat{\beta}=1.5232)$, which means that the solution of the equations exists and is unique.

Step 4. Now we trace the log-likelihood profile with respect to the parameters, see the right part of Figure 1 that indicates the intersection point maximizes the log-likelihood function of the parameters $\alpha$ and $\beta$. 
Step 5. The previous steps indicate that the MLEs of the parameters, $\alpha$ and $\beta$ exist and are unique.

Algorithm 1 is also used for various combinations of parameters with varying sample sizes; in addition, the MLEs of the parameters are found to exist and be unique.

\subsection{Estimation based on product spacings}

The MPS method was proposed by Cheng and Amin (1983). This method is based on an idea that the differences (spacings) of the consecutive points should be identically distributed. The difference is defined as

$$
D_{i}=\int_{x_{(i-1)}}^{x_{(i)}} f(x, \alpha, \beta) d x, \quad i=1,2, \ldots, n+1,
$$

where $F\left(x_{(0)}, \alpha, \beta\right)=0$ and $F\left(x_{(n+1)}, \alpha, \beta\right)=1$. The MPS estimators $\hat{\alpha}_{\mathrm{PS}}$ and $\hat{\beta}_{\mathrm{PS}}$ of $\alpha$ and $\beta$ are obtained by maximizing the geometric mean of the differences. The geometric mean of the differences is given as

$$
G=\sqrt[n+1]{\prod_{i=1}^{n+1} D_{i}}
$$

Substituting (1.3) in the equation above and taking logarithm, we have

$$
\log G(\alpha, \beta)=\frac{1}{n+1} \sum_{i=1}^{n+1} \log \left[\left(1+\frac{\beta}{1+\beta} x_{(i-1)}^{\alpha}\right) e^{-\beta x_{(i-1)}^{\alpha}}-\left(1+\frac{\beta}{1+\beta} x x_{(i)}^{\alpha}\right) e^{-\beta x_{(i)}^{\alpha}}\right] .
$$

The MPS estimators $\hat{\alpha}_{\mathrm{PS}}$ and $\hat{\beta}_{\mathrm{PS}}$ of $\alpha$ and $\beta$ can be obtained by maximizing the $\log G(\alpha, \beta)$ in (3.7).

\subsection{Least squares estimators (LSE)}

Let $x_{(1)}, x_{(2)}, \ldots, x_{(n)}$ be the ordered sample of size $n$ follow the PLD. Then, the expectation

$$
E\left[F\left(X_{(i)}\right)\right]=\frac{i}{n+1}, \quad i=1,2, \ldots, n .
$$

The LSEs $\hat{\alpha}_{\mathrm{LS}}$ and $\hat{\beta}_{\mathrm{LS}}$ of $\alpha$ and $\beta$ are obtained by minimizing

$$
Z(\alpha, \beta)=\sum_{i=1}^{n}\left(F\left(x_{(i)}\right)-\frac{i}{n+1}\right)^{2} .
$$

By substituting the cdf of the PLD in (3.9), we have

$$
Z(\alpha, \beta)=\sum_{i=1}^{n}\left(\frac{n-i+1}{n+1}-\left(1+\frac{\beta}{1+\beta} x_{(i)}^{\alpha}\right) e^{-\beta x_{(i)}^{\alpha}}\right)^{2} .
$$

The LSEs $\hat{\alpha}_{\mathrm{LS}}$ and $\hat{\beta}_{\mathrm{LS}}$ of $\alpha$ and $\beta$ can be obtained by minimizing the $Z(\alpha, \beta)$ in (3.10). The nonlinear equations can be routinely solved using Newton's method. There are many subroutines in R-software such as optim( ) and $\mathrm{nlm}$ ( ) that are tailored to solve the non-linear optimization problems. We recommend the use of the $\mathrm{nlm}($ ) command to optimize the above problems. 


\section{Bayes estimation of the parameters}

In Bayesian set-up, unknown parameters involved in any model are considered to be random variables instated of fixed constants. This idea is logical since the parameters of any population cannot remain the same throughout the study. The variation in the parameters can be incorporated by assuming prior distributions of unknown parameters. Here, we assume that the parameters $\alpha$ and $\beta$ follow independent gamma prior distributions of the following forms:

$$
\begin{array}{ll}
\pi_{1}(\alpha)=\frac{a_{1}^{b_{1}}}{\Gamma\left(b_{1}\right)} \alpha^{b_{1}-1} e^{-a_{1} \alpha}, & \alpha>0, a_{1}, b_{1}>0, \\
\pi_{2}(\beta)=\frac{a_{2}^{b_{2}}}{\Gamma\left(b_{2}\right)} \beta^{b_{2}-1} e^{-a_{2} \beta}, & \beta>0, a_{2}, b_{2}>0,
\end{array}
$$

where $a_{1}, b_{1}, a_{2}$, and $b_{2}$ are called hyper-parameters. The joint prior distribution of $\alpha$ and $\beta$ is given by

$$
\pi(\alpha, \beta)=\frac{a_{1}^{b_{1}} a_{2}^{b_{2}}}{\Gamma\left(b_{1}\right) \Gamma\left(b_{2}\right)} \alpha^{b_{1}-1} \beta^{b_{2}-1} e^{-a_{1} \alpha-a_{2} \beta} .
$$

The joint posteriors of $\alpha$ and $\beta$ is obtained as follows,

$$
\pi(\alpha, \beta \mid \underline{x})=K \frac{\alpha^{n-b_{1}-1} \beta^{2 n-b_{2}-1}}{(1+\beta)^{n}} \prod_{i=1}^{n}\left(1+x_{i}^{\alpha}\right) \prod_{i=1}^{n} x_{i}^{\alpha-1} e^{-\beta\left(\sum_{i=1}^{n} x_{i}^{\alpha}+a_{2}\right)-a_{1} \alpha},
$$

where $K$ is the normalizing constant. Bayes estimates of the parameters under the squared error loss are the means of their respective marginal posteriors. Thus, Bayes estimators of $\alpha$ and $\beta$ are respectively given by

$$
\begin{aligned}
& \hat{\alpha}_{\text {Bayes }}=K \int_{0}^{\infty} \int_{0}^{\infty} \frac{\alpha^{n-b_{1}} \beta^{2 n-b_{2}-1}}{(1+\beta)^{n}} \prod_{i=1}^{n}\left(1+x_{i}^{\alpha}\right) \prod_{i=1}^{n} x_{i}^{\alpha-1} e^{-\beta\left(\sum_{i=1}^{n} x_{i}^{\alpha}+a_{2}\right)-a_{1} \alpha} d \alpha d \beta, \\
& \hat{\beta}_{\text {Bayes }}=K \int_{0}^{\infty} \int_{0}^{\infty} \frac{\alpha^{n-b_{1}-1} \beta^{2 n-b_{2}}}{(1+\beta)^{n}} \prod_{i=1}^{n}\left(1+x_{i}^{\alpha}\right) \prod_{i=1}^{n} x_{i}^{\alpha-1} e^{-\beta\left(\sum_{i=1}^{n} x_{i}^{\alpha}+a_{2}\right)-a_{1} \alpha} d \alpha d \beta .
\end{aligned}
$$

The above expressions are not easy to calculate analytically; therefore, we propose the use of Lindley's approximation and MCMC techniques to obtain Bayes estimates for the parameters.

\subsection{Lindley's approximation}

In 1980, Lindley provided asymptotic solution for the ratio of two integrals generally encountered in Bayesian estimation (Lindley, 1980). Many authors have proposed to use Lindley's approximation to obtain Bayes estimates of the parameters for various lifetime distributions and references cited (Sharma et al., 2015, 2016b). The ratio of integrals occurs in Bayesian analysis is given by

$$
I(\underline{x})=E(u(\Theta) \mid \underline{x})=\frac{\int u(\Theta) \exp \{L(\Theta)+\rho(\Theta)\} d \Theta}{\int \exp \{L(\Theta)+\rho(\Theta)\} d \Theta}
$$


where $\Theta=\left\{\theta_{1}, \theta_{2}, \ldots, \theta_{m}\right\}, u(\Theta)=$ any function of $\Theta, L(\Theta)=$ log-likelihood function, and $\rho(\Theta)=$ $\log$ of joint prior of $\Theta$. For large sample and under some regularity conditions, the above expression can be approximated by the following expansion

$$
I(\underline{x}) \approx u+\frac{1}{2} \sum_{i=1}^{m} \sum_{j=1}^{m}\left(u_{i j}+2 u_{i} \rho_{j}\right) \sigma_{i j}+\frac{1}{2} \sum_{i=1}^{m} \sum_{j=1}^{m} \sum_{k=1}^{m} \sum_{l=1}^{m} L_{i j k} u_{l} \sigma_{i j} \sigma_{k l} .
$$

If $u(\alpha, \beta)=\alpha$, then $u_{1}=1, u_{2}=u_{12}=u_{21}=u_{22}=u_{11}=0$. Therefore, the Bayes estimate of $\alpha$ is then defined as:

$$
\hat{\alpha}_{\mathrm{LD}}=\hat{\alpha}_{\mathrm{ML}}+\sigma_{11} \rho_{1}+\sigma_{12} \rho_{2}+0.5\left\{L_{111} \sigma_{11}^{2}+3 L_{112} \sigma_{11} \sigma_{12}+L_{222} \sigma_{12} \sigma_{22}\right\} .
$$

If $u(\alpha, \beta)=\beta$, then $u_{2}=1, u_{1}=u_{12}=u_{21}=u_{22}=u_{11}=0$. Therefore, the Bayes estimate of $\beta$ can be calculated by the following formula:

$$
\hat{\beta}_{\mathrm{LD}}=\hat{\beta}_{\mathrm{ML}}+\sigma_{21} \rho_{1}+\sigma_{22} \rho_{2}+0.5\left\{L_{222} \sigma_{22}^{2}+L_{112}\left(\sigma_{11} \sigma_{22}+2 \sigma_{12}^{2}\right)+L_{111} \sigma_{11} \sigma_{12}\right\}
$$

\subsection{Markov chain Monte Carlo (MCMC) methods}

Lindley's approximation method is unfeasible for higher dimension models as well as inefficient for a sampler sample size. In such cases, the MCMC iterative methods are found very useful. These methods produce a Markov chain of values that constitute sample draws from posterior distribution; consequently, a sample based inference is drawn. The Gibbs sampler and Metropolis-Hastings (MH) algorithm are basic MCMC methods and frequently used in Bayesian statistics (Gelfand and Smith, 1990; Hastings, 1970). To implement the Gibbs algorithm, the full condition distributions of the parameters $\alpha$ and $\beta$ are given by

$$
\begin{gathered}
\pi_{11}(\alpha \mid \beta, \underline{x}) \propto \alpha^{n-b_{1}-1} \exp \left[\alpha\left(\sum_{i=1}^{n} \log \left(x_{i}\right)-a_{1}\right)-\beta\left(\sum_{i=1}^{n} x_{i}^{\alpha}\right)\right] \prod_{i=1}^{n}\left(1+x_{i}^{\alpha}\right), \\
\pi_{22}(\beta \mid \alpha, \underline{x}) \propto \frac{\beta^{2 n-b_{2}-1}}{(1+\beta)^{n}} e^{-\beta\left(\sum_{i=1}^{n} x_{i}^{\alpha}+a_{2}\right)}
\end{gathered}
$$

respectively. The full conditional distributions are not in standard distributional forms; therefore, we propose the use of $\mathrm{MH}$ algorithm to draw the random sample from the full conditionals. A hybrid algorithm of Gibbs and $\mathrm{MH}$ samplers consists of the following steps:

Step 1. Set initial values $\alpha^{0}$ and $\beta^{0}$ for $\alpha$ and $\beta$, respectively.

Step 2. Using initial values $\alpha^{0}$ and $\beta^{0}$, generate candidate points $\left\{\alpha^{c}, \beta^{c}\right\}$ respectively from the proposal densities $q_{1}\left(\alpha^{c} \mid \alpha^{0}\right)$ and $q_{2}\left(\beta^{c} \mid \beta^{0}\right)$, where $q\left(\Theta^{c} \mid \Theta^{0}\right), \Theta=\{\alpha, \beta\}$ is the probability of returning a value of $\Theta^{c}$ given a previous value of $\Theta^{0}$. Here, we propose the use of asymptotic distributions of MLEs as proposal densities.

Step 3. Generate a uniform variate on range 0 to 1 , i.e., $u \sim U(0,1)$.

Step 4. Calculate Hastings-ratio using candidate point $\alpha_{c}$ and previous point $\alpha^{0}$ as given by

$$
\rho_{1}\left(\alpha^{c} \mid \alpha^{0}\right)=\left(\frac{\pi_{11}\left(\alpha^{c} \mid \beta^{0}, \underline{\boldsymbol{x}}\right) q_{1}\left(\alpha^{0} \mid \alpha^{c}\right)}{\pi_{11}\left(\alpha^{0} \mid \beta^{0}, \underline{\boldsymbol{x}}\right) q_{1}\left(\alpha^{c} \mid \alpha^{0}\right)}\right) .
$$


Step 5. Accept the candidate point as

$$
\alpha= \begin{cases}\alpha^{c}, & \text { if } u \leq \min \left(1, \rho_{1}\left(\alpha^{c} \mid \alpha^{0}\right)\right), \\ \alpha^{0}, & \text { otherwise }\end{cases}
$$

and set $\alpha^{0}=\alpha$.

Step 6. Now using the current point $\alpha^{0}$, calculate Hastings-ratio for the parameter $\beta$ as given by

$$
\rho_{2}\left(\beta^{c} \mid \beta^{0}\right)=\left(\frac{\pi_{22}\left(\beta^{c} \mid \alpha^{0}, \underline{\boldsymbol{x}}\right) q_{2}\left(\beta^{0} \mid \beta^{c}\right)}{\pi_{22}\left(\beta^{0} \mid \alpha^{0}, \underline{\boldsymbol{x}}\right) q_{2}\left(\beta^{c} \mid \beta^{0}\right)}\right) .
$$

Step 7. Accept the candidate point as

$$
\beta= \begin{cases}\beta^{c}, & \text { if } u \leq \min \left(1, \rho_{2}\left(\beta^{c} \mid \beta^{0}\right)\right), \\ \beta^{0}, & \text { otherwise }\end{cases}
$$

and $\operatorname{set} \beta^{0}=\beta$.

Step 8. Repeat Steps 2-9, $M(=10,000)$ times and obtain posterior sample of size $M$ for the parameters $\alpha$ and $\beta$.

Step 9. Bayes estimates of $\alpha$ and $\beta$ under squared error loss, can be obtained as the mean of the simulated samples from their posteriors. Thus, the formulae to obtain Bayes estimates of $\alpha$ and $\beta$ are given by $\hat{\alpha}_{\mathrm{MC}}=1 /\left(M-M_{0}\right) \sum_{i=M_{0}}^{M} \alpha_{i}$ and $\hat{\beta}_{\mathrm{MC}}=1 /\left(M-M_{0}\right) \sum_{i=M_{0}}^{M} \beta_{i}$. Here, $M_{0}$ (burn-in period) is taken to be 1,000 .

\section{Comparison of the proposed estimators}

In this section, we study the behavior of the proposed estimator for the PLD parameters $\alpha$ and $\beta$ with varying sample sizes and different combinations of parameters. The performances of the estimators are compared based on the mean squared error (MSE) using simulated samples. For this purpose, we simulate the random samples of different sizes from PLD using the inversion method; please refer to Sharma et al. (2016a) for the algorithm and R-codes used for this purpose. For each generated sample, we compute the proposed estimates for the PLD parameters. We repeat the process 1,000 times to average the long run performance of the proposed estimators; in addition, the average estimates along with MSEs are also recorded. Under Bayesian estimation, we consider the $\operatorname{Gamma}\left(a_{1}, b_{1}\right)$ and $\operatorname{Gamma}\left(a_{2}, b_{2}\right)$ prior for the unknown parameters $\alpha$ and $\beta$, respectively. We take prior to mean equal to the guess (true) values of the parameters with variance. In this study, we take the prior variances equal to 1 (small) and 100 (large) known as Gamma-I and Gamma-II priors, respectively. Under non-informative prior, the hyper-parameters are taken to be zero.

We generate 10,000 MCMC draws from the posterior using MCMC algorithms. The asymptotic posterior is taken to be the proposal density for simulation and MLEs are considered initial guesses under the MH algorithm. We also study convergences of the MCMC chains using cumulative means and trace plots; consequently, it is found that the MCMC chains converge rapidly to stationary distributions. Furthermore, we take every second point from the chains so that autocorrelation can be reduced to a certain level. Tables 1 and 2 summarizes the simulation results. However, we only show 
Table 1: Average estimates (in first row of each cell) and MSE (in second row of each cell) of the classical estimators for $n=30$

\begin{tabular}{|c|c|c|c|c|c|c|c|}
\hline \multirow[b]{2}{*}{$\alpha$} & \multirow[b]{2}{*}{$\beta$} & \multicolumn{3}{|c|}{$\alpha$} & \multicolumn{3}{|c|}{$\beta$} \\
\hline & & $\begin{array}{c}\text { MLE } \\
\hat{\alpha}_{\mathrm{ML}}\end{array}$ & $\begin{array}{l}\mathrm{LSE} \\
\hat{\alpha}_{\mathrm{LS}}\end{array}$ & $\begin{array}{c}\text { MPS } \\
\hat{\alpha}_{\mathrm{PS}}\end{array}$ & $\begin{array}{c}\text { MLE } \\
\hat{\beta}_{\mathrm{ML}}\end{array}$ & $\begin{array}{c}\text { LSE } \\
\hat{\beta}_{\mathrm{LS}}\end{array}$ & $\begin{array}{c}\text { MPS } \\
\hat{\beta}_{\text {PS }}\end{array}$ \\
\hline \multirow{2}{*}{1} & 1 & $\begin{array}{l}1.048 \\
0.026\end{array}$ & $\begin{array}{l}0.992 \\
0.032\end{array}$ & $\begin{array}{l}0.949 \\
0.022\end{array}$ & $\begin{array}{l}1.009 \\
0.032\end{array}$ & $\begin{array}{l}1.021 \\
0.032\end{array}$ & $\begin{array}{l}1.031 \\
0.028\end{array}$ \\
\hline & 4 & $\begin{array}{l}1.053 \\
0.029 \\
\end{array}$ & $\begin{array}{l}0.993 \\
0.036 \\
\end{array}$ & $\begin{array}{l}0.947 \\
0.025\end{array}$ & $\begin{array}{l}4.384 \\
1.181 \\
\end{array}$ & $\begin{array}{l}4.124 \\
1.473\end{array}$ & $\begin{array}{l}3.850 \\
0.642\end{array}$ \\
\hline \multirow{2}{*}{2} & 1 & $\begin{array}{l}2.096 \\
0.102\end{array}$ & $\begin{array}{l}1.983 \\
0.124\end{array}$ & $\begin{array}{l}1.898 \\
0.088 \\
\end{array}$ & $\begin{array}{l}1.009 \\
0.032\end{array}$ & $\begin{array}{l}1.021 \\
0.032 \\
\end{array}$ & $\begin{array}{l}1.031 \\
0.028 \\
\end{array}$ \\
\hline & 4 & $\begin{array}{l}2.105 \\
0.119\end{array}$ & $\begin{array}{l}1.987 \\
0.143\end{array}$ & $\begin{array}{l}1.894 \\
0.099\end{array}$ & $\begin{array}{l}4.384 \\
1.181 \\
\end{array}$ & $\begin{array}{l}4.124 \\
1.473\end{array}$ & $\begin{array}{l}3.850 \\
0.642 \\
\end{array}$ \\
\hline \multirow{2}{*}{3} & 1 & $\begin{array}{l}3.144 \\
0.229 \\
\end{array}$ & $\begin{array}{l}2.975 \\
0.279 \\
\end{array}$ & $\begin{array}{l}2.846 \\
0.198 \\
\end{array}$ & $\begin{array}{l}1.009 \\
0.032 \\
\end{array}$ & $\begin{array}{l}1.022 \\
0.032 \\
\end{array}$ & $\begin{array}{l}1.031 \\
0.028 \\
\end{array}$ \\
\hline & 4 & $\begin{array}{l}3.158 \\
0.269\end{array}$ & $\begin{array}{l}2.979 \\
0.321 \\
\end{array}$ & $\begin{array}{l}2.841 \\
0.224 \\
\end{array}$ & $\begin{array}{l}4.384 \\
1.181 \\
\end{array}$ & $\begin{array}{l}4.124 \\
1.473\end{array}$ & $\begin{array}{l}3.851 \\
0.641 \\
\end{array}$ \\
\hline \multirow{2}{*}{4} & 1 & $\begin{array}{l}4.192 \\
0.408\end{array}$ & $\begin{array}{l}3.966 \\
0.498\end{array}$ & $\begin{array}{l}3.795 \\
0.352\end{array}$ & $\begin{array}{l}1.009 \\
0.032\end{array}$ & $\begin{array}{l}1.021 \\
0.032\end{array}$ & $\begin{array}{l}1.031 \\
0.028\end{array}$ \\
\hline & 4 & $\begin{array}{l}4.211 \\
0.478\end{array}$ & $\begin{array}{l}3.973 \\
0.570\end{array}$ & $\begin{array}{l}3.787 \\
0.398\end{array}$ & $\begin{array}{l}4.384 \\
1.181\end{array}$ & $\begin{array}{l}4.124 \\
1.474\end{array}$ & $\begin{array}{l}3.850 \\
0.642\end{array}$ \\
\hline
\end{tabular}

MLE = maximum likelihood estimate; LSE = least squares estimate; MPS = maximum product spacings.

Table 2: Average Bayes estimate and mean squared error of parameters $\alpha$ and $\beta$ with varying parameters and $n=30$

\begin{tabular}{|c|c|c|c|c|c|c|c|c|c|c|c|c|}
\hline \multirow{2}{*}{$\alpha, \beta$} & \multicolumn{4}{|c|}{ Non-informative } & \multicolumn{4}{|c|}{ Gamma-1 } & \multicolumn{4}{|c|}{ Gamma-2 } \\
\hline & $\hat{\alpha}_{\mathrm{LD}}$ & $\hat{\beta}_{\mathrm{LD}}$ & $\hat{\alpha}_{\mathrm{MC}}$ & $\hat{\beta}_{\mathrm{MC}}$ & $\hat{\alpha}_{\mathrm{LD}}$ & $\hat{\beta}_{\mathrm{LD}}$ & $\hat{\alpha}_{\mathrm{MC}}$ & $\hat{\beta}_{\mathrm{MC}}$ & $\hat{\alpha}_{\mathrm{LD}}$ & $\hat{\beta}_{\mathrm{LD}}$ & $\hat{\alpha}_{\mathrm{MC}}$ & $\hat{\beta}_{\mathrm{MC}}$ \\
\hline \multirow{2}{*}{1,1} & 1.039 & 1.018 & 1.041 & 0.999 & 1.036 & 1.018 & 1.038 & 0.999 & 1.038 & 1.018 & 1.041 & 0.999 \\
\hline & 0.025 & 0.032 & 0.025 & 0.031 & 0.023 & 0.029 & 0.023 & 0.029 & 0.024 & 0.032 & 0.025 & 0.031 \\
\hline \multirow{2}{*}{1,3} & 1.064 & 3.298 & 1.025 & 3.109 & 1.045 & 3.155 & 1.017 & 3.050 & 1.064 & 3.297 & 1.025 & 3.108 \\
\hline & 0.036 & 0.656 & 0.026 & 0.358 & 0.027 & 0.203 & 0.022 & 0.176 & 0.036 & 0.649 & 0.026 & 0.355 \\
\hline \multirow{2}{*}{2,1} & 2.039 & 1.008 & 2.082 & 0.999 & 2.027 & 1.009 & 2.072 & 1.001 & 2.039 & 1.008 & 2.082 & 0.999 \\
\hline & 0.079 & 0.033 & 0.100 & 0.031 & 0.063 & 0.030 & 0.082 & 0.028 & 0.079 & 0.033 & 0.099 & 0.031 \\
\hline \multirow{2}{*}{2,3} & 2.243 & 3.490 & 2.051 & 3.109 & 2.194 & 3.337 & 2.029 & 3.048 & 2.242 & 3.488 & 2.050 & 3.108 \\
\hline & 0.287 & 1.331 & 0.103 & 0.358 & 0.193 & 0.548 & 0.077 & 0.172 & 0.286 & 1.321 & 0.103 & 0.354 \\
\hline \multirow{2}{*}{3,1} & 2.738 & 1.035 & 3.123 & 0.999 & 2.699 & 1.039 & 3.095 & 1.003 & 2.737 & 1.035 & 3.122 & 0.999 \\
\hline & 0.124 & 0.031 & 0.225 & 0.031 & 0.123 & 0.027 & 0.152 & 0.027 & 0.124 & 0.030 & 0.224 & 0.031 \\
\hline \multirow{2}{*}{3,3} & 3.722 & 3.863 & 3.076 & 3.109 & 3.622 & 3.693 & 3.035 & 3.045 & 3.721 & 3.861 & 3.075 & 3.108 \\
\hline & 1.818 & 3.499 & 0.233 & 0.358 & 1.269 & 1.979 & 0.139 & 0.168 & 1.812 & 3.482 & 0.231 & 0.354 \\
\hline
\end{tabular}

the tables for sample size 30 due to space restrictions. The performances of the proposed estimators can be described as:

- All the estimators show the property of consistency, i.e., the MSE decreases as sample size increases.

- In all the considered cases (classical and Bayesian), the MSE of the estimator(s) of $\alpha$ and $\beta$ increases as parametric value of $\alpha$ and $\beta$ increase while $n$ and other parameters are maintained as fixed.

- In classical estimation, the MSE of (all) the estimator(s) of $\alpha$ increases as the parametric value $\beta$ increases, while sample size $n$ and parameter $\alpha$ are maintained as fixed. However, no specific 
Table 3: LRT for fitting LD and PLD based on real data sets

\begin{tabular}{|c|c|c|c|c|}
\hline & \multicolumn{2}{|c|}{ JSPI } & \multicolumn{2}{|c|}{ CSDA } \\
\hline & LD & PLD & LD & PLD \\
\hline$\hat{\alpha}$ & - & 1.1822 & - & 1.3864 \\
\hline$\hat{\beta}$ & 0.1455 & 0.0898 & 0.1708 & 0.0660 \\
\hline KS & 0.0953 & 0.0506 & 0.1614 & 0.0544 \\
\hline$p$-value & 0.0126 & 0.4733 & 0.0000 & 0.5184 \\
\hline $\log (\ell)$ & 951.1600 & 943.0200 & 718.9000 & 695.8600 \\
\hline LRT & \multirow{2}{*}{\multicolumn{2}{|c|}{$\begin{array}{l}16.2878 \\
5.44 \mathrm{E}-05\end{array}$}} & \multicolumn{2}{|c|}{46.0688} \\
\hline$p$-value & & & \multicolumn{2}{|c|}{$1.14 \mathrm{E}-11$} \\
\hline Decision & \multicolumn{2}{|c|}{ Reject $H_{0}$} & & \\
\hline
\end{tabular}

JSPI = Journal of Statistical Planning and Inference; CSDA = Computational Statistics and Data Analysis; LD = Lindley distribution; PLD = power Lindley distribution; KS = Kolmogorov-Smirnov; LRT = likelihood ratio test.

pattern has been found in the MSE of the estimator $\beta$ with increasing $\alpha$ when $n$ and $\beta$ are maintained as fixed.

- Product spacings estimators are more efficient than MLE and LSE in classical estimation. In general, the MSEs of the above three estimators can be ordered as MSE (MPS) $<$ MSE (MLE) $<$ MSE (LSE).

- Bayes estimators obtained with the MCMC technique are superior to Bayes estimators obtained using Lindley's approximation.

- Bayes estimators obtained using Lindley's approximation are worse if $n$ is small and with large parametric values of $\alpha$ and $\beta$, i.e., in case of small sample size, the Lindley's approximation technique cannot be recommended.

- Bayes estimators obtained under the assumption of the Gamma priors are superior to those obtained under the non-informative priors.

- The MSE of Bayes estimators increase as prior variance increases.

- Bayes estimators obtained using the MCMC technique under the assumption of gamma priors with small variances are the most efficient estimators among all proposed Bayes estimators.

\section{Application: waiting time to publication in statistical journal}

The waiting time of the research article to be accepted (WTA) for its publication in a journal is the time interval between the submission date and the acceptance date of the article. The analysis of such data is especially significant for young researchers who are in the early age of their career and need to protect and give a height to their career. There are many reasons by which the article may take more time for publication such as a busy schedule for reviewers and editors, a long queue of articles. The WTA can be governed through probability law because it is random and cannot be pre-determined. This section proposes the use of the PLD for the WTA data.

We collected the data on WTA for research articles published in JSPI and CSDA in 2012. The WTA is computed as

$$
\text { WTA }=\text { Acceptance date }- \text { Submission date } \text {. }
$$




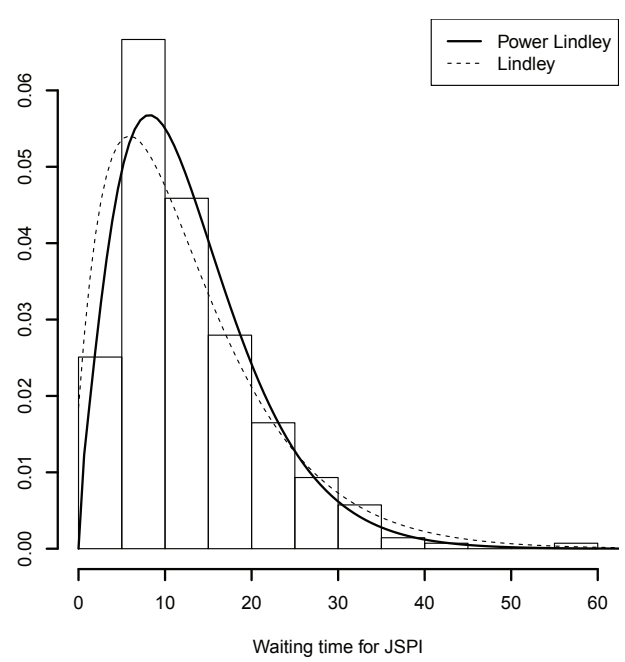

(a)

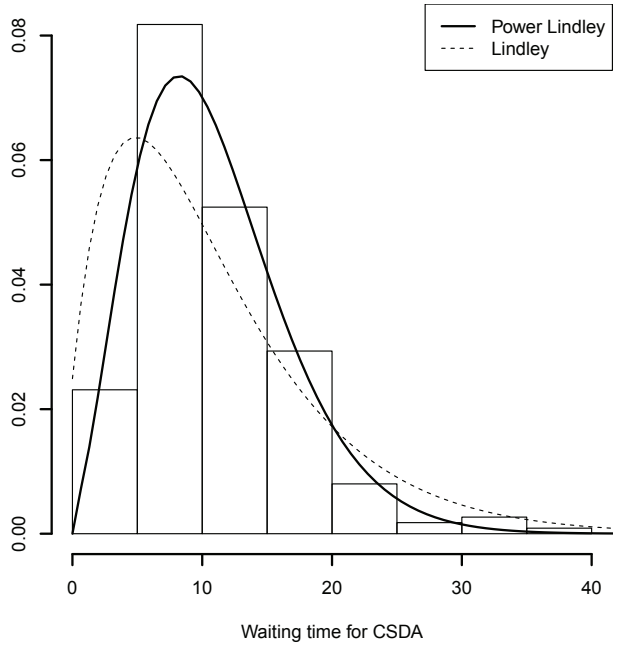

(b)

Figure 2: Fitted density function of power Lindley distribution and Lindley distribution for waiting times (in months) data for JSPI (a) and CSDA (b). JSPI = Journal of Statistical Planning and Inference; CSDA = Computational Statistics and Data Analysis.

It is of interest to compare the applicability of the PLD with its sub-model for given sets of WTA data. For this purpose, we use likelihood ratio test to test the null hypothesis $H_{0}: \alpha=1(X \sim \operatorname{LD}(\beta))$ against $H_{1}: \alpha \neq 1(X \sim \operatorname{PLD}(\alpha, \beta))$. Table 3 shows that the PLD fits well with the data over its sub-model. The same can also be observed from the fitted densities plots presented in Figures 2 (a) and (b). We next access the goodness-of-fit of the PLD with two other parameter distributions defined by the following density functions.

- Generalized exponential distribution (GED), $f(x)=\alpha \lambda e^{-\lambda x}\left(1-e^{-\lambda x}\right)^{\alpha-1}, x>0, \alpha, \lambda>0$,

- Weibull distribution (WD), $f(x)=\theta p x^{p-1} e^{-\theta x^{p}}, x>0, \alpha, p>0$,

- Gamma distribution (GD), $f(x)=\left(\theta^{\alpha} / \Gamma(\alpha)\right) x^{\alpha-1} e^{-\theta x}, x>0, \alpha, \theta>0$,

- Flexible Weibull (FW), $f(x)=\left(\alpha+\beta / x^{2}\right) \exp (\alpha x-\beta / x) \exp (-\exp (\alpha x-\beta / x)), x>0, \alpha, \beta>0$,

- Weighted Lindley (WL), $f(x)=\left[\theta^{c} /((\theta+c) \Gamma(c))\right] x^{c-1}(1+x) e^{-\theta x}, x>0, \theta, c>0$,

- Inverse Weibull (IW), $f(x)=\alpha \lambda x^{-\alpha-1} e^{-\lambda x^{-\alpha}}, x>0, \alpha, \lambda>0$,

- Generalized inverted exponential distribution (GIED), $f(x)=\left(\alpha \lambda / x^{2}\right) \exp (-\lambda / x)[1-\exp (-\lambda / x)]^{\alpha-1}$, $x>0, \alpha, \lambda>0$.

We use chi-square $\left(\chi^{2}\right)$, Kolmogorov-Smirnov (KS) goodness-of-fit tests to compare the fitting of the distributions. In many situations, we may have more than one model that provides a reasonable fit to the data according to the $\left(\chi^{2}\right)$ and KS tests at a given level of significance. In such cases, model compatibility can be compared using Akaike information criterion (AIC) and Bayesian information criterion (BIC). The fitting summary of the models for both data sets obtained from JSPI and CSDA 
Table 4: Maximum likelihood estimates (SE) and goodness-of-fit statistics for the considered models based on the waiting times obtained from the Journal of Statistical Planning and Inference

\begin{tabular}{cccccccc}
\hline \hline Model & Estimates (SE) & KS & $p$-value & $-\log L$ & AIC & BIC & Rank \\
\hline PLD & $\hat{\alpha}=1.182(0.047), \hat{\beta}=0.089(0.012)$ & 0.051 & 0.473 & 943.02 & 1890.03 & 1897.29 & 1 \\
GLD & $\hat{\alpha}=1.461(0.136), \hat{\lambda}=0.175(0.009)$ & 0.039 & 0.778 & 943.26 & 1890.52 & 1897.78 & 2 \\
GED & $\hat{\alpha}=2.478(0.228), \hat{\lambda}=0.129(0.008)$ & 0.043 & 0.582 & 948.53 & 1901.06 & 1908.32 & 6 \\
WD & $\hat{p}=1.617(0.081), \hat{\theta}=0.013(0.003)$ & 0.057 & 0.323 & 947.81 & 1899.61 & 1906.87 & 5 \\
GD & $\hat{\alpha}=2.306(0.183), \hat{\theta}=0.179(0.016)$ & 0.039 & 0.677 & 947.42 & 1898.84 & 1906.10 & 4 \\
FW & $\hat{\alpha}=0.043(0.002), \hat{\beta}=2.502(0.276)$ & 0.622 & 0.000 & 1149.05 & 2302.10 & 2309.36 & 8 \\
WL & $\hat{c}=1.576(0.176), \hat{\theta}=0.192(0.016)$ & 0.040 & 0.739 & 944.23 & 1892.45 & 1899.71 & 3 \\
IW & $\hat{\alpha}=0.699(0.023), \hat{\lambda}=3.642(0.230)$ & 0.248 & 0.000 & 1119.76 & 2243.53 & 2250.79 & 7 \\
GIED & $\hat{\alpha}=0.638(0.052), \hat{\lambda}=2.542(0.258)$ & 0.324 & 0.000 & 1196.11 & 2396.21 & 2403.47 & 9 \\
\hline \hline
\end{tabular}

SE = standard error; KS = Kolmogorov-Smirnov; AIC = Akaike information criterion; BIC = Bayesian information criterion; PLD = power Lindley distribution; GLD = generalized Lindley distribution; GED = generalized exponential distribution; WD = Weibull distribution; GD = Gamma distribution; FW = flexible Weibull; $\mathrm{WL}=$ weighted Lindley; IW = inverse Weibull; GIED = generalized inverted exponential distribution .

Table 5: The $\chi^{2}$ statistic, observed frequency $(\mathrm{O})$ and expected frequency for considered distributions based on WTA obtained from JSPI and CSDA

\begin{tabular}{rcrrrrrrrrr}
\hline \hline & $\mathrm{O}$ & \multicolumn{1}{c}{ PLD } & \multicolumn{1}{c}{ GLD } & \multicolumn{1}{c}{ GED } & \multicolumn{1}{c}{ WD } & \multicolumn{1}{c}{ GD } & \multicolumn{1}{c}{ FW } & \multicolumn{1}{c}{ WL } & IW & GIED \\
\hline \multirow{3}{*}{ JSPI } & Total 279 & 278.963 & 278.880 & 278.710 & 278.980 & 278.870 & 278.990 & 278.900 & 226.730 & 242.460 \\
& $\chi^{2}$ & 13.810 & 11.560 & 12.530 & 19.220 & 12.520 & 230.010 & 12.540 & 204.660 & 308.760 \\
& $p$-value & 0.387 & 0.564 & 0.484 & 0.116 & 0.485 & 0.000 & 0.483 & 0.000 & 0.000 \\
\hline \multirow{3}{*}{ CSDA } & Total 225 & 224.832 & 224.381 & 223.865 & 224.906 & 224.489 & 224.812 & 224.541 & 171.439 & 192.041 \\
& $\chi^{2}$ & 16.916 & 13.007 & 13.764 & 21.995 & 13.609 & 170.086 & 13.982 & 201.984 & 258.808 \\
& $p$-value & 0.031 & 0.112 & 0.088 & 0.005 & 0.092 & 0.000 & 0.082 & 0.000 & 0.000 \\
\hline \hline
\end{tabular}

WTA = waiting time of research article to be accepted; JSPI = Journal of Statistical Planning and Inference; CSDA = Computational Statistics and Data Analysis; O = observed frequency; PLD = power Lindley distribution; GLD = generalized Lindley distribution; GED = generalized exponential distribution; WD = Weibull distribution; GD = Gamma distribution; FW = flexible Weibull; WL = weighted Lindley; IW = inverse Weibull; GIED = generalized inverted exponential distribution .

are summarized in Tables 4-6. From the KS test, it can be observed that FW, IW, and GIED distributions do not show their compatibility to the data sets; however, PLD, GLD, WL, GED, GD, and WD do fit the data sets. More than one distribution provides a reasonable fit; therefore, we need to use AIC and BIC to select the best possible distribution. We find that PLD provides the best fit for the WTA data as it shows the lowest AIC and BIC among other distributions. Table 7 presents the estimates based on the WTA and the PLD parameters. The mean WTA for the research articles published in CSDA and JSPI are 10.87 and 12.88, respectively. This means that the research articles take 10-11 and 12-13 months (on an average) for the acceptance of their publication in CSDA and JSPI, respectively.

\section{Conclusions}

In this paper, PLD is considered with its properties and applications. In a classical set-up, three different methods of estimation (MLE, MPS, and LSE) were discussed to estimate unknown PLD parameters. It is found that the MPS estimators outperform MLE and LSE in terms of their MSEs. The Bayesian set-up considers the parameters to follow the independent gamma prior distributions. Bayes estimators are constructed under the assumption of quadratic loss function. Lindley's approximation and MCMC techniques are used since explicit derivations for Bayes estimators cannot be obtained. It is observed that the MCMC techniques provide good approximation to the posterior as well as provide 
Table 6: Maximum likelihood estimates (SE), KS statistics, and the corresponding $p$-value for the considered models based on waiting times obtained from Computational Statistics and Data Analysis

\begin{tabular}{cccccccc}
\hline \hline Model & Estimates (SE) & KS & $p$-value & $-\log L$ & AIC & BIC & Rank \\
\hline PLD & $\hat{\alpha}=1.387(0.062), \hat{\beta}=0.066(0.011)$ & 0.055 & 0.512 & 695.83 & 1395.66 & 1396.37 & 1 \\
GLD & $\hat{\alpha}=2.194(0.246), \hat{\lambda}=0.243(0.014)$ & 0.045 & 0.751 & 696.13 & 1396.26 & 1396.96 & 2 \\
GED & $\hat{\alpha}=3.636(0.401), \hat{\lambda}=0.183(0.012)$ & 0.052 & 0.575 & 701.55 & 1407.13 & 1407.81 & 6 \\
WD & $\hat{p}=1.903(0.116), \hat{\theta}=0.008(0.003)$ & 0.071 & 0.204 & 701.52 & 1407.04 & 1407.74 & 5 \\
GD & $\hat{\alpha}=3.197(0.287), \hat{\theta}=0.294(0.028)$ & 0.051 & 0.604 & 699.76 & 1403.53 & 1404.24 & 4 \\
FW & $\hat{\alpha}=0.057(0.003), \hat{\beta}=3.070(0.343)$ & 0.406 & 0.000 & 874.23 & 1752.46 & 1753.17 & 8 \\
WL & $\hat{c}=2.451(0.282), \hat{\theta}=0.307(0.028)$ & 0.051 & 0.606 & 697.62 & 1399.23 & 1399.93 & 3 \\
IW & $\hat{\alpha}=0.733(0.025), \hat{\lambda}=3.754(0.263)$ & 0.286 & 0.000 & 863.01 & 1730.02 & 1730.72 & 7 \\
GIED & $\hat{\alpha}=0.766(0.073), \hat{\lambda}=3.062(0.334)$ & 0.345 & 0.000 & 920.39 & 1844.79 & 1845.49 & 9 \\
\hline \hline
\end{tabular}

SE = standard error; KS = Kolmogorov-Smirnov; AIC = Akaike information criterion; BIC = Bayesian information criterion; PLD = power Lindley distribution; GLD = generalized Lindley distribution; GED = generalized exponential distribution; WD = Weibull distribution; GD = Gamma distribution; FW = flexible Weibull; WL = weighted Lindley; IW = inverse Weibull; GIED = generalized inverted exponential distribution.

Table 7: Classical and Bayes estimates of the parameters of power Lindley distribution for waiting times data sets of CSDA and JSPI

\begin{tabular}{|c|c|c|c|c|c|}
\hline \multirow{2}{*}{ Method } & & \multicolumn{2}{|c|}{ CSDA } & \multicolumn{2}{|c|}{ JSPI } \\
\hline & & $\bar{\alpha}$ & $\beta$ & $\alpha$ & $\beta$ \\
\hline \multirow{3}{*}{ Classical } & MLE & 1.386 & 0.066 & 1.182 & 0.089 \\
\hline & LSE & 1.431 & 0.062 & 1.215 & 0.085 \\
\hline & MPS & 1.358 & 0.070 & 1.159 & 0.095 \\
\hline \multirow{3}{*}{ Lindley distribution } & Non-inf & 1.350 & 0.073 & 1.156 & 0.097 \\
\hline & Gamma-1 & 1.350 & 0.073 & 1.156 & 0.097 \\
\hline & Gamma-2 & 1.350 & 0.073 & 1.156 & 0.097 \\
\hline \multirow{3}{*}{ Markov chain Monte Carlo } & Non-inf & 1.393 & 0.064 & 1.186 & 0.088 \\
\hline & Gamma-1 & 1.393 & 0.064 & 1.186 & 0.088 \\
\hline & Gamma-2 & 1.393 & 0.064 & 1.186 & 0.088 \\
\hline
\end{tabular}

JSPI = Journal of Statistical Planning and Inference; CSDA = Computational Statistics and Data Analysis; MLE = maximum likelihood estimate; LSE = least squares estimate; MPS = maximum product spacings; Non-inf = non-informative.

more efficient estimates for the parameters than those obtained using Lindley's approximation. The PLD is applied to model the waiting times for research articles to be accepted for publication in statistical journals and it is found that the PLD reasonably fits the data better than other competing distributions. Finally, we can conclude that the PLD has nice properties with closed forms for the survival and hazard functions as well as provides a better model than other competing distributions such as Weibull, gamma, and IW.

\section{Appendix}

\section{Lemma 1. Let}

$$
I(\alpha, \beta, n, x)=\int_{x}^{\infty}\left(1+u^{\alpha}\right) u^{\alpha+n-1} e^{-\beta u^{\alpha}} d u
$$

We have,

$$
I(\alpha, \beta, n, x)=\frac{1}{\alpha \beta} e^{-\beta x^{\alpha}} x^{n} \sum_{j=0}^{\infty} \frac{(-1)^{j}}{\left(\beta x^{\alpha}\right)^{j}}\left(b_{1 j}+b_{2 j} x^{\alpha}\right),
$$


where $b_{10}=b_{20}=1, b_{1 j}=\left(1-a_{1}\right)\left(2-a_{1}\right) \cdots\left(j-a_{1}\right), \quad b_{2 j}=\left(1-a_{2}\right)\left(2-a_{2}\right) \cdots\left(j-a_{2}\right), \quad a_{1}=$ $n / \alpha+1, a_{2}=n / \alpha+2$.

Proof: One can simply prove this Lemma using the definition of incomplete gamma function.

Lemma 2. Let

$$
G(a, b, c, A, B)=\int_{0}^{\infty} t^{a-1} \exp \left(-b t^{A}-c t^{B}\right) d t
$$

Then, we have

$$
\begin{aligned}
G(a, b, c, A, B) & =\frac{1}{A B b^{\frac{a}{A}}} \frac{1}{2 \pi i} \int_{\gamma-i \infty}^{\gamma+i \infty} \Gamma\left(\frac{a+s}{A}\right) \Gamma\left(\frac{s}{B}\right)\left(c^{\frac{1}{B}} b^{\frac{1}{A}}\right)^{-s} d t \\
& =\frac{1}{A B b^{\frac{a}{A}}} H_{0,2}^{2,0}\left[\left.c^{\frac{1}{B}} b^{\frac{1}{A}}\right|_{\left(0, \frac{1}{B}\right)\left(\frac{a}{A}, \frac{1}{A}\right)}\right],
\end{aligned}
$$

where $H$ denotes the H-function Mathai et al. (2010) and is defined as

$$
H_{p, q}^{m, n}\left[z z_{\left(b_{1}, B_{1}\right) \cdots\left(b_{q}, B_{q}\right)}^{\left(a_{1}, A_{1}\right) \cdots\left(a_{p}, A_{p}\right)}\right]=\frac{1}{2 \pi i} \int_{\gamma-i \infty}^{\gamma+i \infty} \frac{\prod_{j=1}^{m} \Gamma\left(b_{j}+B_{j} s\right) \prod_{j=1}^{n} \Gamma\left(1-a_{j}-A_{j} s\right)}{\prod_{j=m+1}^{q} \Gamma\left(1-b_{j}-B_{j} s\right) \prod_{j=n+1}^{p} \Gamma\left(a_{j}+A_{j} s\right)} z^{-s} d s,
$$

$m, n, p, q \in N_{0}$ with $0 \leq n \leq p, 1 \leq m \leq q ; A_{j}, B_{j} \in R_{+}$and $a_{j}, b_{j} \in R$ or $C$.

Proof: Consider, the integral of the form

$$
g(u)=\int_{0}^{\infty} \frac{1}{v} f_{1}\left(\frac{u}{v}\right) f_{2}(v) d v .
$$

By the convolution property of Millen transformation (Mathai et al., 2010), we have

$$
M_{g}(s)=M_{f_{1}}(s) M_{f_{2}}(s),
$$

where $M_{g}(s)$ denotes the Millen transformation of function $g$ and is defined as

$$
M_{g}(s)=\int_{0}^{\infty} u^{s-1} g(u) d u, \quad G(a, b, c, A, B)=\int_{0}^{\infty} \frac{1}{t} \underbrace{t^{a} e^{-b t^{A}}}_{f_{1}} \underbrace{e^{-c t^{B}}}_{f_{2}} d t,
$$

then $M_{G}(s)=[1 / A][\Gamma((a+s) / A)] / b^{(a+s) / A} \times[1 / B][\Gamma(s / B)] / c^{s / B}$. The inverse Millen transformation of above expression yields the integral

$$
\begin{aligned}
G(a, b, c, A, B) & =\frac{1}{A B b^{\frac{a}{A}}} \frac{1}{2 \pi i} \int_{\gamma-i \infty}^{\gamma+i \infty} \Gamma\left(\frac{a+s}{A}\right) \Gamma\left(\frac{s}{B}\right)\left(c^{\frac{1}{B}} b^{\frac{1}{A}}\right)^{-s} d t \\
& =\frac{1}{A B b^{\frac{a}{A}}} H_{0,2}^{2,0}\left[\left.c^{\frac{1}{B}} b^{\frac{1}{A}}\right|_{\left.\left(0, \frac{1}{B}\right)\left(\frac{a}{A}, \frac{1}{A}\right)\right] .}\right.
\end{aligned}
$$

\section{Lemma 3.}

$$
I(a, b)=\int_{0}^{\infty} y^{a-1} \log (y) e^{-b y} d y=\frac{\Gamma(a)}{b^{a}}[\psi(a)-\log (b)], \quad a, b>0,
$$

where $\psi(y)=(d / d y) \ln \Gamma(y)$ is a digamma function.

Proof: See, Gradshteyn and Ryzhik (2007, p 573) integral. 


\section{References}

Abdous B and Berred A (2005). Mean residual life estimation, Journal of Statistical Planning and Inference, 132, 3-19.

Ali S, Aslam M, and Kazmi SMA (2013). A study of the effect of the loss function on Bayes Estimate, posterior risk and hazard function for Lindley distribution, Applied Mathematical Modelling, 37, 6068-6078.

Al-Mutairi DK, Ghitany ME, and Kundu D (2013). Inferences on stress-strength reliability from Lindley distributions, Communications in Statistics - Theory and Methods, 42, 1443-1463.

Cheng RCH and Amin NAK (1983). Estimating parameters in continuous univariate distributions with a shifted origin, Journal of the Royal Statistical Society Series B (Methodological), 45, 394-403.

Gelfand AE and Smith AF (1990). Sampling-based approaches to calculating marginal densities, Journal of the American Statistical Association, 85, 398-409.

Ghitany ME, Al-Mutairi DK, and Aboukhamseen SM (2015). Estimation of the reliability of a stressstrength system from power Lindley distributions, Communications in Statistics - Simulation and Computation, 44, 118-136.

Ghitany ME, Al-Mutairi DK, Balakrishnan N, and Al-Enezi LJ (2013). Power Lindley distribution and associated inference, Computational Statistics and Data Analysis, 64, 20-33.

Ghitany ME, Atieh B and Nadarajah S (2008). Lindley distribution and its application, Mathematics and Computers in Simulation, 78, 493-506.

Govil KK and Aggarwal KK (1983). Mean residual life function for normal, gamma and lognormal densities, Reliability Engineering, 5, 47-51.

Gradshteyn IS and Ryzhik IM (2007). Tables of Integrals, Series, and Products (7th ed), Academic Press, New York.

Gui W and Chen M (2016). Parameter estimation and joint confidence regions for the parameters of the generalized Lindley distribution, Mathematical Problems in Engineering, 2016, 1-13.

Gupta RD and Kundu D (2009). Introduction of shape/skewness parameter(s) in a probability distribution, Journal of Probability and Statistical Science, 7, 153-171.

Hastings WK (1970). Monte Carlo sampling methods using Markov chains and their applications, Biometrika, 57, 97-109.

Krishna H and Kumar K (2011). Reliability estimation in Lindley distribution with progressively type II right censored sample, Mathematics and Computers in Simulation, 82, 281-294.

Lindley DV (1958). Fiducial distributions and Bayes' theorem, Journal of the Royal Statistical Society Series B (Methodological), 20, 102-107.

Lindley DV (1980). Approximate Bayesian methods, Trabajos de Estadistica y de Investigacion Operativa, 31, 223-245.

Mathai AM, Saxena RK, and Haubold HJ (2010). The H-Function: Theory and Applications, Springer Science \& Business Media, New York.

Nadarajah S, Bakouch HS, and Tahmasbi R (2011). A generalized Lindley distribution, Sankhya B, 73, 331-359.

Okwuokenye M and Peace KE (2015). A comparison of size and power of tests of hypotheses on parameters based on two generalized Lindley distributions, Communications for Statistical Applications and Methods, 22, 233-239.

Sharma VK, Singh SK, Singh U, and Agiwal V (2015). The inverse Lindley distribution: a stressstrength reliability model with application to head and neck cancer data, Journal of Industrial and Production Engineering, 32, 162-173. 
Sharma VK, Singh SK, Singh U, and Merovci F (2016a). The generalized inverse Lindley distribution: a new inverse statistical model for the study of upside-down bathtub data, Communications in Statistics - Theory and Methods, 45, 5709-5729.

Sharma VK, Singh SK, Singh U, and Ul-Farhat K (2016b). Bayesian estimation on interval censored Lindley distribution using Lindley's approximation, International Journal of System Assurance Engineering and Management, https://doi.org/10.1007/s13198-016-0528-x.

Singh B, Gupta PK, and Sharma VK (2014a). Parameter estimation of power Lindley distribution under hybrid censoring, Journal of Statistics Applications $\mathcal{E}$ Probability Letters: An International Journal, 1, 95-104.

Singh SK, Singh U, and Sharma VK (2013). Expected total test time and Bayesian estimation for generalized Lindley distribution under progressively Type-II censored sample where removals follow the beta-binomial probability law, Applied Mathematics and Computation, 222, 402-419.

Singh SK, Singh U, and Sharma VK (2014b). Bayesian estimation and prediction for the generalized Lindley distribution under asymmetric loss function, Hacettepe Journal of Mathematics and Statistics, 43, 661-678. 
